agent for phosphate control. Nicotinic acid therapy was started after a 2-week washout period, and continued for 12 weeks. The initial daily dose was $375 \mathrm{mg}$ but was increased every 2 weeks to 500,1,000, 1,500 and finally $2,000 \mathrm{mg}$, as tolerated.

The target dose of $>1,000 \mathrm{mg}$ nicotinic acid daily was tolerated by 17 patients, whose mean dose was $1,470 \pm 110 \mathrm{mg}$ daily. After 12 weeks of treatment (completed by 11 patients), average serum phosphate levels had decreased significantly from $2.3 \mathrm{mmol} / \mathrm{l}(7.2 \mathrm{mg} / \mathrm{dl})$ to $1.9 \mathrm{mmol} / \mathrm{l}(5.9 \mathrm{mg} / \mathrm{dl} ; P=0.015$ [K/DOQl target $5.5 \mathrm{mg} / \mathrm{dl}]$ ). Concentrations of serum calcium and parathyroid hormone were unaffected. A marked increase in $\mathrm{HDL}$ cholesterol level from 1.04 to $1.53 \mathrm{mmol} / \mathrm{l}$ ( 40 to $59 \mathrm{mg} / \mathrm{dl} ; P=0.0005$ ) was also observed, but triglyceride concentrations did not change significantly. Adverse effects of nicotinic acid included flushing and diarrhea.

Original article Müller D et al. (2007) Niacin lowers serum phosphate and increases HDL cholesterol in dialysis patients. Clin J Am Soc Nephrol 2: 1249-1254

\section{Mathematical modeling of pathogen transmission control in a dialysis unit}

Patients undergoing dialysis are at high risk of infection, with viruses and antibiotic-resistant bacteria being particularly problematic. Devising a model to predict the impact of various infection control measures is difficult because of the spatial heterogeneity of dialysis units, small patient populations, and the uncertainty of knowing which patients are infectious.

Hotchkiss et al. report the development of a new Monte Carlo simulation tool that takes the above difficulties into account. With literaturebased estimates of pathogen transmissibility, prevalence and diagnostic uncertainty used as variables, the model was employed to predict the effect of three facility-level control strategies on dissemination of pathogens through a medium-sized dialysis unit (46 patients per day).

As expected, the model showed that environmental decontamination, particularly of dialysis chairs, could markedly reduce the risk of nosocomial dissemination. Temporal segregation was another effective means of theoretically reducing transmission. Scheduling patients with suspected infection for dialysis sessions later in the day and decontaminating the unit thoroughly before the next treatment sessions are simple, low-cost interventions that should minimize nosocomial transmission in dialysis units.

The authors conclude that this new model could be used to determine how best to deploy scarce resources to improve patient safety.

Original article Hotchkiss JR et al. (2007) Analyzing pathogen transmission in the dialysis unit: time for a (schedule) change? Clin J Am Soc Nephrol 2: 1176-1185

\section{Accurate therapeutic monitoring of tacrolimus by use of dried blood spot sampling}

Currently, monitoring of levels of the immunosuppressant tacrolimus requires repeated venous blood draws, which are costly, and inconvenient for patients and staff. Cheung et al. investigated whether measurement of tacrolimus levels in dried blood spots by use of high-performance liquid chromatography tandem-mass spectrometry (HPLC-MS) is a viable alternative.

Duplicate venous samples, and simultaneous fingerprick samples drawn by 36 stable renal transplant recipients, were obtained at different time points (108 sets of samples in total). One venous sample from each set was analyzed immediately at a hospital in Hong Kong. The other venous samples and the dried blood spots were sent to the University of Maastricht in The Netherlands for analysis.

Tacrolimus levels measured from fingerprick blood samples through use of HPLC-MS showed an excellent correlation (coefficient of determination $\left.r^{2}=0.96 ; P<0.0001\right)$ with those obtained from aliquots of venous blood by use of the same method in the same center. On average, dried samples had been stored for 40 days. There was a similarly high correlation between the HPLC-MS results obtained at the two centers from venous samples, indicating that blood remained stable after long-distance transport and storage.

The authors have proved the utility of the self-administered fingerprick technique as an alternative to venipuncture for obtaining blood to monitor therapeutic levels of tacrolimus in kidney transplant patients. The convenience 